\title{
Embedding with Vaidya geometry
}

\author{
A. V. Nikolaev ${ }^{1,2, a}{ }_{(\mathbb{O})}$, S. D. Maharaj ${ }^{1, \mathrm{~b}}{ }_{(\mathbb{C})}$ \\ ${ }^{1}$ Astrophysics and Cosmology Research Unit, School of Mathematics, Statistics and Computer Science, University of KwaZulu-Natal, Durban, \\ South Africa \\ ${ }^{2}$ Ulyanovsk State Pedagogical University named after I.N. Ulyanov, Ulyanovsk, Russia
}

Received: 28 February 2020 / Accepted: 11 July 2020 / Published online: 20 July 2020

(C) The Author(s) 2020

\begin{abstract}
The Vaidya metric is important in describing the exterior spacetime of a radiating star and for describing astrophysical processes. In this paper we study embedding properties of the generalized Vaidya metric. We had obtained embedding conditions, for embedding into 5-dimensional Euclidean space, by two different methods and solved them in general. As a result we found the form of the mass function which generates a subclass of the generalized Vaidya metric. Our result is purely geometrical and may be applied to any theory of gravity. When we apply Einstein's equations we find that the embedding generates an equation of state relating the null string density to the null string pressure. The energy conditions lead to particular metrics including the anti/de Sitter spacetimes.
\end{abstract}

\section{Introduction}

The local embedding of a four-dimensional pseudo-Riemannian spacetime can be isometrically embedded in a higher dimensional Euclidean space. A discussion of the embedding equations and embedding conditions are given in Eisenhart [1]. The invariance of the embedding class generates a classification scheme for the exact solutions of the Einstein field equations. Examples of explicit embeddings and solutions of the Einstein field equations which are embeddable are given in the works of Rosen [2], Stephani [3] and Collinson [4]. The conditions for the integrability of the embeddings are determined by the Gauss-Codazzi-Ricci equations. A comprehensive review of explicit embeddings for particular spacetimes and solutions of the Gauss-Codazzi-Ricci equations, for embedding Classes I and II, are contained in Stephani et al. [5]. For Class I spacetimes the embedding space is a 5-dimensional Euclidean space, and for Class II spacetimes the embedding space is a 6-dimensional Euclidean space.

\footnotetext{
a e-mail: ilc@xhns.org

be-mail: maharaj@ukzn.ac.za (corresponding author)
}

An important reason for studying embeddings is that this approach may lead to new exact solutions of the field equations. In addition to applications in general relativity, embeddings are important for studies in extrinsic gravity, strings and membranes, and brane world cosmologies are pointed out by Pavsic [6].

Recently embeddings have been intensively studied in relativistic astrophysics and applied to models of compact objects. The Gauss-Codazzi-Ricci equations in spherically symmetric spacetimes provide a necessary and sufficient condition for the embedding to be Class I. For the embedding Karmarkar [7] found the explicit restrictions on the Riemann tensor components. Physically reasonable static stellar structures via embedding have been found by Maurya and Govender [8], Maurya et al. [9,10], and Singh et al. [11]. Kuhfittig [12] used embeddings to study charged wormholes and flat rotation curves in spiral galaxies. The case of traversable wormholes was considered in Ref. [13] for a complete solution with both redshift and shape functions. Several other studies of stellar models with the embedding Class I condition are contained in the works [14-29]. A general algorithm to describe embeddable anisotropic compact stellar models was discovered by Murad [30]. These astrophysical applications apply to static spherically symmetric fields. An interesting nonstatic embeddable stellar interior was produced for the first time by Naidu et al. [31] which satisfies the Karmarkar condition. In this nonstatic model the star is radiating and the exterior atmosphere is given by the Vaidya spacetime.

We comment on embeddings and stellar exterior metrics which appear to be linked. The Schwarzschild exterior metric represents the vacuum exterior of a star. It is embeddable in a 6-dimensional space; consequently the Schwarzschild exterior is Class II embeddable as first established by Kasner [32]. The Vaidya spacetime may be used to model the exterior of a radiating object. The generalized Vaidya spacetime may be embedded in a 5-dimensional Euclidean space; this spacetime is Class I embeddable for particular mass func- 
tions as we establish in this paper. However it is interesting to observe that the conventional Vaidya metric is not Class I embeddable.

The generalized Vaidya geometry [33] is essential in describing the physical features of a radiating star in general relativity. The generalized Vaidya spacetime has applications in relativistic astrophysics as pointed out in recent investigations. As an example, we refer to the study of Maharaj et al. [34] who showed that the generalized Vaidya spacetime may be interpreted as a superposition of a null fluid and a null string fluid for the exterior of a radiating star. As a second example, we note that Naidu et al. [31], generated a specific model of a relativistic radiating star for a particular spacetime metric matching to the generalized Vaidya spacetime which satisfies the Karmarkar embedding condition. It is therefore possible to directly link the embedding condition to the evolution of a spherically symmetric radiating star.

A comprehensive analysis of the embedding of the generalized Vaidya spacetime may lead to new insights in the gravitational behavior of the system. It is therefore important to study the embedding properties of this geometry which is the objective of this paper. In Sect. 2 we review the generalized Vaidya geometry and the Einstein field equations. Two different approaches of embedding the generalized Vaidya metric into the 5-dimensional Euclidean geometry are considered in Sect. 3. The condition on the mass function is found as a nonlinear differential equation. In Sect. 4 we show that the embedding is characterised by an equation of state. The equations for a radiating star are considered in Sect. 6. Some concluding remarks are given in Sect. 6. The details of the embedding equations are given in the Appendix. For convenience we set $c=8 \pi G=1$ in this paper.

\section{Vaidya geometry}

The Vaidya metric can be written in the form

$$
\begin{aligned}
d s^{2}= & -\left(1-\frac{2 m(v)}{r}\right) d \nu^{2}-2 d \nu d r \\
& +r^{2}\left(d \theta^{2}+\sin ^{2} \theta d \phi^{2}\right)
\end{aligned}
$$

where the function $m(v)$ is called the mass function. It is important in describing the exterior spacetime of a radiating star. For recent examples of radiating stellar structures see the works of Govinder and Govender [35], Reddy et al. [36] and Das et al. [37]. The Einstein field equations for the matter distribution

$$
T_{\mu \nu}=\varepsilon k_{\mu} k_{\nu}
$$

where $\varepsilon$ is the density of the outgoing null radiation and $k^{\mu} k_{\mu}=0$, reduces to

$\varepsilon=-2 \frac{m_{v}}{r^{2}}$

the function $m(v)$ represents the mass of the star as measured by an observer at infinity.

The generalized Vaidya metric has the form

$$
\begin{aligned}
d s^{2}= & -\left(1-\frac{2 m(v, r)}{r}\right) d \nu^{2}-2 d \nu d r, \\
& +r^{2}\left(d \theta^{2}+\sin ^{2} \theta d \phi^{2}\right)
\end{aligned}
$$

where the mass function depends both on $v$ and $r$. This metric is important in describing the exterior of a radiating star with a generalized atmosphere. The junction conditions at the stellar surface with the metric (4) where generated by Maharaj et al. [34]. The physics of generalized Vaidya atmosphere, and exact radiating models were investigated by Govender et al. [38], Naidu et al. [39] and Brassel et al. [40]. A consistent matter distribution for the spacetime (4) is

$T_{\mu \nu}=\varepsilon k_{\mu} k_{\nu}+(\rho+P)\left(k_{\mu} l_{\nu}+k_{\nu} l_{\mu}\right)+P g_{\mu \nu}$,

where $l^{\mu} l_{\mu}=0$ and $l^{\mu} k_{\mu}=-1$. In the above $\rho$ is the null string energy density and $P$ is the null string pressure. The matter tensor (5) describes an atmosphere which is a superposition of null dust (with $\varepsilon$ ), and null string fluid (with $\rho$ and $P$ ). This interpretation was first given in the works of $[41,42]$. The Einstein field equations then reduce to

$$
\begin{aligned}
& \varepsilon=-2 \frac{\dot{m}}{r^{2}}, \\
& \rho=2 \frac{m^{\prime}}{r^{2}}, \\
& P=-\frac{m^{\prime \prime}}{r},
\end{aligned}
$$

with energy momentum (5). In the above dots and primes refer to differentiation with respect to $v$ and $r$, respectively.

\section{Embedding condition}

The embedding condition of a 4-dimensional Riemannian metric into an pseudo-Euclidean 5-dimensional metric requires it to be Class I (see [5]). To achieve the embedding we need to solve the Gauss-Codazzi-Ricci equations. There is no known general method to solve these equations, and therefore the embedding has been demonstrated only in special cases. Sometimes the approach of finding a transformation directly embedding the Riemannian metric into the higher dimensional Euclidean metric is possible. We show that both approaches work for the generalized Vaidya metric. 


\subsection{Direct embedding approach}

We seek to embed the curved generalized Vaidya metric (4) into a flat 5-dimensional metric

$d s^{2}=-\left(d x_{0}\right)^{2}+\left(d x_{1}\right)^{2}+\left(d x_{2}\right)^{2}+\left(d x_{3}\right)^{2}+\left(d x_{4}\right)^{2}$,

where $\left(x_{\alpha}\right)=\left(x_{0}, x_{1}, x_{2}, x_{3}, x_{4}\right)$. Karmarkar [7] has suggested an algorithm which we follow, and assume

$x_{2}=r \sin \theta \cos \phi$,

$x_{3}=r \sin \theta \sin \phi$,

$x_{4}=r \cos \theta$.

Then (7) becomes

$d s^{2}=-\left(d x_{0}\right)^{2}+\left(d x_{1}\right)^{2}+d r^{2}+r^{2}\left(d \theta^{2}+\sin ^{2} \theta d \phi^{2}\right)$.

Then from (4) and (9) we have

$$
\begin{aligned}
& -\left(1-\frac{2 m(v, r)}{r}\right) d v^{2}-2 d v d r-d r^{2} \\
& =-\left(d x_{0}\right)^{2}+\left(d x_{1}\right)^{2}
\end{aligned}
$$

which places a restriction on the mass function $m(\nu, r)$, and shows that the left hand side of the metric has to be flat.

A necessary and sufficient condition for metric being flat is that the Gaussian curvature be zero. The general Gaussian curvature is given by

$K=\frac{P_{h i j k} p^{h} q^{i} p^{j} q^{k}}{\left(\gamma_{h j} \gamma_{i k}-\gamma_{h k} \gamma_{i j}\right) p^{h} q^{i} p^{j} q^{k}}$,

where $P_{h i j k}$ is the Riemann tensor in two dimensions, $p^{h}$ and $q^{i}$ are vectors in the 2 -surface $\left(\gamma_{i j}\right.$ is the metric in this space) according to Weatherburn [43]. In the above the tensor indices are defined on the 2 -sphere and $i=1,2$. According to theorem of Schur [44], the Riemannian curvature of a space is independent of the orientation chosen, thus (11) simplifies to the following

$P_{h i j k}=K\left(\gamma_{h j} \gamma_{i k}-\gamma_{h k} \gamma_{i j}\right)$.

For the left hand side of (10) we have only one independent Riemann tensor component

$P_{1212}=\frac{r^{2} m^{\prime 2}+2 r m m^{\prime}-3 m^{2}-2 r^{2} m m^{\prime \prime}}{2 m r^{3}}$.

Thus with (12) and (13) the embedding condition for the generalized Vaidya metric is

$2 r^{2} m m^{\prime \prime}-r^{2} m^{\prime 2}-2 r m m^{\prime}+3 m^{2}=0$,

which is a nonlinear equation in $m$.

\subsection{Gauss-Coddazzi-Ricci equations}

The Gauss-Coddazzi-Ricci equations for Class I embedding have the general form

$R_{\gamma \delta}^{\mu \nu}= \pm\left(b_{\gamma}^{\mu} b_{\delta}^{v}-b_{\delta}^{\mu} b_{\gamma}^{\nu}\right)$,

$b_{\nu ; \gamma}^{\mu}=b_{\gamma ; v}^{\mu}$,

according to Stephani et al. [5] and $b_{\mu \nu}$ is a symmetric tensor. Note that the quantities $R_{\mu \nu \delta \gamma}$ and $b_{\mu \nu}$ are defined in the 4-dimensional Riemannian manifold. In this case the tensor indices are defined on the 4-dimensional spacetime manifold and $\mu=0,1,2,3$. In the above (15a) corresponds to the Gauss equations and (15b) corresponds to the Codazzi equations. The Ricci equations are identically satisfied for Class I embedding. Effectively the embedding equations (15a) and (15b) will place restrictions on the components of the Riemann tensor $R_{\mu \nu \delta \gamma}$.

The embedding restriction for a spherically symmetric spacetime to be Class I was first found by Karmarkar [7] which is a necessary condition; the sufficient condition for this result to hold was established by Pandey and Sharma [45]. We now find the restrictions on the Riemann curvature for the generalized Vaidya metric (4). The Gauss-CodazzRicci equations imply the result

$R_{1220}=-\frac{\sqrt{-R_{1010} R_{2323}}}{\sin \theta}$,

which is an equation in terms of our independent Riemann tensor components. We can also write (16) in the equivalent form

$R_{1220} R_{1330}+R_{1010} R_{2323}=0$,

since $R_{1220} \sin ^{2} \theta=R_{1330}$. The derivation of (17) is given in the Appendix. We observe that the values of the Riemann tensor components arising in (16) are

$R_{1220}=\frac{r m^{\prime}-m}{r}$,

$R_{1010}=-\frac{r^{2} m^{\prime \prime}-2 r m^{\prime}+2 m}{r^{3}}$,

$R_{2323}=2 r m \sin ^{2} \theta$.

Substituting (18a)-(18c) into (16) yields

$2 r^{2} m m^{\prime \prime}-r^{2} m^{\prime 2}-2 r m m^{\prime}+3 m^{2}=0$.

Observe that the embedding condition (19) is the same as (14) obtained by the direct method.

We should point out that the restriction (17) can also be obtained from the Karmarkar embedding condition. For a spherically symmetric line element it is well known that 
embedding leads to the following equation

$R_{1220} R_{1330}+R_{1010} R_{2323}-R_{1212} R_{3030}=0$,

relating various components of the Riemann tensor. For the generalized Vaidya spacetime (4) we have

$R_{1212}=0$,

which is a further restriction. We observe that (20) become (17) when (21) holds.

\subsection{The mass function}

The embedding into 5-dimensional flat spacetime has generated the condition (14) on the mass function $m(v, r)$. This is a nonlinear partial differential equation. However it can be integrated as the $v$-dependence is not explicit. We can rewrite (14) in the from

$2 r^{2} \frac{m^{\prime \prime}}{m}-r^{2} \frac{m^{\prime 2}}{m^{2}}-2 r \frac{m^{\prime}}{m}+3=0$.

If we let $z=\frac{m^{\prime}}{m}$ then (22) becomes

$z^{\prime}+\frac{1}{2} z^{2}-\frac{1}{r} z=-\frac{3}{2 r^{2}}$

which is a Riccati equation. In general Riccati equations do not have a general solution. However in our case (23) admits the particular solution $z=\frac{1}{r}$. Consequently the transformation

$z=\frac{1}{r}+\frac{1}{u}$

leads to the linear equation

$u^{\prime}=\frac{1}{2}$.

This has the solution

$u=\frac{1}{2} r+\frac{1}{2} C_{1}(v)$,

where $\frac{1}{2} C_{1}(v)$ is a function of integration. In terms of the variable $z$ we have

$z=\frac{1}{r}+\frac{2}{r+C_{1}(v)}$.

Using $z=\frac{m^{\prime}}{m}$ we can integrate (27) to obtain

$\ln m=\ln r+\ln \left(r+C_{1}(v)\right)^{2}+\ln C_{2}(v)$,

where $C_{2}(v)$ is a second function of integration. We can write this result compactly as

$m(v, r)=r C_{2}(v)\left(r+C_{1}(v)\right)^{2}$.

Hence the embedding condition for the generalized Vaidya metric leads to a restriction on the mass function $m(v, r)$ given by (29). Note that the condition (29) does not allow for the mass function to depend on the variable $v$ only with $m(v)$.
Hence the conventional Vaidya metric (1) is not embeddable in 5-dimensional flat spacetime but particular forms of the generalized Vaidya metric are embeddable. It is interesting to observe that the dependence in the retarded time coordinate $v$ is arbitrary in equation (29). The dependence in the radial coordinate $r$ is fully known and the mass function has the form

$m(v, r)=C_{2}(v) r^{3}+2 C_{1}(v) C_{2}(v) r^{2}+C_{2}(v) C_{1}^{2}(v) r,(30)$

which is a cubic function in $r$.

Embedding leads to an explicit functional form for the mass function $m(v, r)$. The condition (29) was obtained from the Gauss and Codazzi equations which are necessary and sufficient conditions for the metric to be Class I embeddable. Thus we can state the following theorem:

Theorem 1 The necessary and sufficient condition for the generalized Vaidya metric to be Class I embeddable is that the mass function must have the form

$m(v, r)=r g(v)(r+f(v))^{2}$,

where $g(v)$ and $f(v)$ are arbitrary functions.

\section{Application to general relativity}

Theorem 1 was obtained using only geometry and no field equations were assumed. Consequently Theorem 1 holds in general relativity or any modified theory of gravity with relevant field equations. It will be interesting to consider the effect of this embedding in various modified theories in future work. As a first step we demonstrate an application to general relativity.

Using (31) and the field equations we obtain

$$
\begin{aligned}
& \varepsilon=-\frac{2(r+f)((r+f) \dot{g}+2 g \dot{f})}{r}, \\
& \rho=\frac{2 g(r+f)(3 r+f)}{r^{2}}, \\
& P=-\frac{2 g(3 r+2 f)}{r},
\end{aligned}
$$

for the Class I embeddable generalized Vaidya spacetime. Equations (32a)-(32c) solve the Einstein field equations with the two free functions $g(v)$ and $f(v)$.

It is clear from the above equations that it is impossible to write an equation of state of the from $P=P(\rho)$ in general. However it is possible to find a particular relationship between $P$ and $\rho$. We can eliminate the coordinate $r$ from (32b) and (32c) to obtain

$\rho=\frac{1}{8 g} P^{2}-\frac{1}{2} P-\frac{3}{2} g$, 
which is of the form $F(\rho, P, v)=0$. If we take $g=\frac{1}{2} C$, a constant, then we have

$\rho=\frac{1}{4 C} P^{2}-\frac{1}{2} P-\frac{3}{4} C$,

which is an equation of state of the form $F(\rho, P)=0$. Hence the embedding of the generalized Vaidya spacetime into 5dimensional Euclidean space is characterized by the equation of state (34). Hence the null string density $\rho$ can be written as an explicit function of the null string pressure $P$ for the embeddable generalized Vaidya metric. As the equation of state in (34) is different from previous studies, our model will exhibit qualitatively different gravitational behaviour. Note that several equations of state of the form $P=P(\rho)$ exist in the generalized Vaidya spacetime (in the absence of embedding). These have been widely studied by Husain [41], Wang and $\mathrm{Wu}[46]$, and Glass and Krisch [42,47]. Many of the models with an equation of state can be used to describe diffusive processes in the stellar exterior as the null string density then satisfies the diffusion equation. Dawood and Ghosh [48] and Ghosh and Dawood [49] discovered a family of exact solutions characterising a type II fluid which contain the familiar dynamical black hole solutions. Brassel et al. [40,50] considered various equations of state for collapsing stars in relation to the cosmic censorship hypothesis. We emphasize that the relationship (34) arises from the Einstein field equations; the energy conditions may place further restrictions.

We now consider the energy conditions following the approach of Wang and $\mathrm{Wu}$ [46] for the generalized Vaidya metric grouped by fluid type. The energy conditions for a type II fluid $(m=m(v, r))$ are

1. The weak and strong energy conditions:

$\varepsilon \geq 0, \quad \rho \geq 0, \quad P \geq 0, \quad(\varepsilon \neq 0)$.

2. The dominant energy condition:

$$
\varepsilon \geq 0, \quad \rho \geq P \geq 0, \quad(\varepsilon \neq 0) .
$$

The energy conditions for type I fluid $(m=m(r))$ are

1. The weak energy condition:

$$
\rho \geq 0, \quad \rho+P \geq 0, \quad(\varepsilon=0) .
$$

2. The strong energy condition:

$$
\rho+P \geq 0, \quad P \geq 0, \quad(\varepsilon=0)
$$

3. The dominant energy condition:

$$
\rho \geq 0, \quad-\rho \leq P \leq \rho, \quad(\varepsilon=0) .
$$

From (32a)-(32c) we see that, without any additional restrictions, the conditions in (35) are violated. Conditions (36) are violated too because of negative pressure $(P<0)$, all other inequalities are satisfied if $\dot{g} \leq 0$ and $\dot{f} \leq 0$. Thus in general $P<0$, for arbitrary functions $g(v)$ and $f(v)$, and embedding provides dark energy type behavior.

It is interesting to note that we can generate type I metrics which are static $(\varepsilon=0$ and $m=m(r))$ and type II metrics ( $\varepsilon \neq 0$ and $m=m(\nu, r)$ ) from embedding. Two of the metrics are well known and third metric appears to be new.

\section{1 de Sitter and anti-de Sitter (type I)}

To reproduce the de Sitter and anti-de Sitter solutions we set

$f=0, \quad g=\frac{\lambda}{6}$,

in (31). As a result we find that

$$
\begin{aligned}
m & =\frac{\lambda}{6} r^{3}, \\
\rho & =-P=\lambda .
\end{aligned}
$$

Thus we regain the case of the generalized Vaidya de Sitter or anti-de Sitter solutions depending on the sign of $g$.

\subsection{Linear equation of state (type II)}

To reproduce the solution with a linear equation of state we set

$f=0$,

in (31). We find that

$\begin{aligned} m & =g r^{3}, \\ \rho & =-P=6 g, \quad \varepsilon=-2 r \dot{g} .\end{aligned}$

We should note that in this case the weak and strong energy conditions (35) as well as dominant energy condition (36) are violated because of negative pressure $(P<0)$ but $\varepsilon$ and $\rho$ are positive if $\dot{g} \leq 0$.

\subsection{Nonlinear equations of state (type I)}

To study this case we should have that the mass function depends only on $r$, namely

$f=$ constant,$g=$ constant.

We then obtain

$$
\begin{aligned}
& m=r g(r+f)^{2}, \\
& \rho=\frac{2 g(r+f)(3 r+f)}{r^{2}}, \quad P=-\frac{2 g(3 r+2 f)}{r},
\end{aligned}
$$


$\rho=\frac{1}{8 g} P^{2}-\frac{1}{2} P-\frac{3}{2} g$,

which represents a Class I embeddable type I fluid in general relativity. The weak energy condition (37) and the dominant energy condition (39) are satisfied if $f \geq 0$. The strong energy condition (38) is violated. The equations of state (45c) has the form $\rho=F(P)$. This result appears to be new and is not shown in studies involving embedding.

Generalized Vaidya metrics have several astrophysical applications. These metrics may be used to model a radiating star, describe superposition of different fluid types and study gravitational collapse as shown by Naidu et al. [39], Maharaj et al. [34] and Brassel et al. [40]. An interesting astrophysical application is to interpret the generalized Vaidya spacetime as a diffusive medium as first shown by Glass and Krisch [42]. Here the component of the energy momentum tensor corresponding to the null string fluid is linked to point particle diffusion. From classical transport theory we consider the diffusion equation in the form

$\dot{\rho}=\frac{D}{r^{2}} \frac{\partial}{\partial r}\left(r^{2} \rho^{\prime}\right)$,

where $D$ is a constant. We now impose the integrability condition for the mass function $\frac{\partial}{\partial \nu} m^{\prime}=\frac{\partial}{\partial r} \dot{m}$ and substitute into field equations (32a)-(32c) to obtain

$\dot{\rho}=-\frac{1}{r^{2}} \frac{\partial}{\partial r}\left(r^{2} \varepsilon\right)$.

We substitute (47) into (46) and obtain after simplification

$\dot{m}=D\left(m^{\prime \prime}-\frac{2}{r} m^{\prime}\right)$.

Hence interpretation of the generalized Vaidya spacetime as a diffusive medium leads to the condition to the mass function. If we substitute above (31) into (48) we obtain the consistency condition

$r(r+f)^{2} \dot{g}+2 r g(r+f) \dot{f}+4 D g f+\frac{2}{r} D g f^{2}=0$,

which is satisfied only if $f=0$ and $\dot{g}=0$. Thus we regain the de Sitter metric (40). Hence we conclude that embedding does place restrictions on the physical features of the system; embedding does not allow the interpretation of the generalized Vaidya spacetime as a diffusive medium.

\section{Radiating stars}

Several models of radiating stars undergoing dissipation in general relativity are known. The interior matter distribution is heat conducting and the exterior spacetime is given by the conventional Vaidya metric. For comprehensive treatments of recent exact solutions for radiating stellar models see Abebe and Maharaj [52], Mohanlal et al. [53] and
Mohamed et al. [54-56]. The generalized Vaidya spacetime may also be used to model a radiating star in general relativity. For this general case the exterior spacetime may be interpreted as a a generalized external atmosphere consisting of a combination two fluids: a null dust fluid and a null string fluid. The interior spacetime remains heat conducting and the exterior spacetime is now given by (4). The relevant model, field equations and junction conditions were developed by Maharaj et al. [34].

The interior spacetime is given by the shear-free metric

$d s^{2}=-A^{2} d t^{2}+B^{2}\left[d r^{2}+r^{2}\left(d \theta^{2}+\sin ^{2} \theta d \phi^{2}\right)\right]$,

where $A(t, r)$ and $B(t, r)$ are the metric functions. The interior matter distribution has the form

$T_{\mu \nu}=(\tilde{\rho}+\tilde{p}) u_{\mu} u_{v}+\tilde{p} g_{\mu \nu}+\tilde{q}_{\mu} u_{\nu}+\tilde{q}_{\nu} u_{\mu}$,

where $\tilde{\rho}$ is the energy density, $\tilde{p}$ is the isotropic pressure, $\tilde{q}_{\mu}$ is the radial heat flux vector and $u^{\mu}=\frac{1}{A} \delta_{0}^{\mu}$ is the comoving fluid four-velocity. The Einstein field equations in the interior of the radiating star have the form

$$
\begin{aligned}
\tilde{\rho}= & 3 \frac{\dot{B}^{2}}{A^{2} B^{2}}-\frac{1}{B^{2}}\left(2 \frac{B^{\prime \prime}}{B}-\frac{B^{\prime 2}}{B^{2}}+\frac{4}{r} \frac{B^{\prime}}{B}\right), \\
\tilde{p}= & \frac{1}{A^{2}}\left(-2 \frac{\ddot{B}}{B}-\frac{\dot{B}^{2}}{B^{2}}+2 \frac{\dot{A}}{A} \frac{\dot{B}}{B}\right), \\
& +\frac{1}{B^{2}}\left(\frac{B^{\prime 2}}{B^{2}}+2 \frac{A^{\prime}}{A} \frac{B^{\prime}}{B}+\frac{2}{r} \frac{A^{\prime}}{A}+\frac{2}{r} \frac{B^{\prime}}{B}\right), \\
\tilde{p}= & -2 \frac{\ddot{B}}{A^{2} B}+2 \frac{\dot{A}}{A^{3}} \frac{\dot{B}}{B}-\frac{\dot{B}^{2}}{A^{2} B^{2}}+\frac{1}{r} \frac{A^{\prime}}{A B^{2}} \\
& +\frac{1}{r} \frac{B^{\prime}}{B^{3}}+\frac{A^{\prime \prime}}{A B^{2}}-\frac{B^{\prime 2}}{B^{4}}+\frac{B^{\prime \prime}}{B^{3}}, \\
\tilde{q}= & -\frac{2}{A B^{2}}\left(-\frac{\dot{B}^{\prime}}{B}+\frac{B^{\prime} \dot{B}}{B^{2}}+\frac{A^{\prime}}{A} \frac{\dot{B}}{B}\right) .
\end{aligned}
$$

The exterior spacetime is given by the generalized Vaidya metric (4):

$$
\begin{aligned}
d s^{2}= & -\left(1-\frac{2 m(\nu, r)}{r}\right) d v^{2}-2 d v d r \\
& +r^{2}\left(d \theta^{2}+\sin ^{2} \theta d \phi^{2}\right)
\end{aligned}
$$

which depends on the mass function $m(v, r)$. The exterior matter distribution has the form

$$
T_{\mu \nu}=\varepsilon k_{\mu} k_{\nu}+(\rho+P)\left(k_{\mu} l_{\nu}+k_{\nu} l_{\mu}\right)+P g_{\mu \nu},
$$

given in (5) for the external matter quantities $\epsilon, \rho$ and $P$. The embedding places a restriction on the form of the mass function $m$.

The line elements (50) and (53) must match at the the stellar surface $\Sigma$. Also the extrinsic curvature has to match for a stable body. Matching generates the junction conditions at the stellar surface $\Sigma$. The resulting important equation, that 
arises in the matching process, that governs the evolution of the radiating star is given by

$$
(\tilde{p}-\tilde{q})_{\Sigma}=\rho_{\Sigma}
$$

This fundamental equation relates the external null string density $\rho$ to the internal pressure $\tilde{p}$ and the heat flux $\tilde{q}$. at the surface of a star with a generalized atmosphere. As $\rho$ is specified by (55) through embedding, and $\tilde{p}, \tilde{q}$ follow from the spherical metric, we observe that (55) is a second order nonlinear partial differential equation. An exact solution to (55) will determine the dynamical evolution of a radiating star with a generalized exterior atmosphere with embedding. Exact solutions to (55) will provide further insight into the process of gravitational collapse. The simpler case when $m=$ $m(v)$ has been mainly studied in the past so that

$$
(\tilde{p}=\tilde{q})_{\Sigma}
$$

Observe that exact solutions of (55) and (56), without embedding, have been found by Govender et al. [38] and Govender [51] for nonadiabatic spherical collapse. As far as we are aware the only embeddable radiating stellar model known was found by Naidu et al. [39] - however this model holds only for the conventional Vaidya model. In future work we will investigate the particular solutions of (55) that are possible when the spacetime is embeddable.

\section{Discussion}

In this research we studied embedding of the generalized Vaidya spacetime into 5-dimensional Euclidean manifold. The conventional Vaidya metric is not Class I embeddable but we proved that particular forms of the generalized Vaidya metric are embeddable. Using two different methods we managed to obtain the embedding condition which is a second order partial differential equation for the mass function. Using a new variable we reduced this equation to the form of a Riccati equation. In general Riccati equations do not have a general solution, but we managed to linearise the embedding equation and solved it in general. It was shown that the embedding condition for the generalized Vaidya metric leads to restriction on the mass function which has arbitrary dependence in the retarded time coordinate and cubic dependence in the radial coordinate. We emphasize that the above calculations were made without assuming any theory of gravity. The result obtained may be applied to a particular theory of gravity such as Einsteins equations or the Einstein-GaussBonnet theory, etc. As an application we applied the obtained result to the Einstein theory. We considered equations of state, the energy conditions, the boundary condition at the stellar surface, the diffusive model of a radiating star. We conclude that the generalized Vaidya spacetime is embeddable with specific form of the mass function and equation of state. The form of the mass function places restrictions on the physical behavior of the system such as allowing for negative pressure. Negative pressure is now a well accepted feature in cosmological models in classical general relativity to explain observations.

Acknowledgements AN and SDM thank the National Research Foundation and the University of KwaZulu-Natal for continuing support. SDM acknowledge that this work is based on research supported by the South African Research Chair Initiative of the Department of Science and Technology and the National Research Foundation.

Data Availability Statement This manuscript has no associated data or the data will not be deposited. [Authors' comment: This is a theoretical study and no experimental data were analyzed during the development of this work. The results apply specifically for this project and they can easily be verified from the information provided.]

Open Access This article is licensed under a Creative Commons Attribution 4.0 International License, which permits use, sharing, adaptation, distribution and reproduction in any medium or format, as long as you give appropriate credit to the original author(s) and the source, provide a link to the Creative Commons licence, and indicate if changes were made. The images or other third party material in this article are included in the article's Creative Commons licence, unless indicated otherwise in a credit line to the material. If material is not included in the article's Creative Commons licence and your intended use is not permitted by statutory regulation or exceeds the permitted use, you will need to obtain permission directly from the copyright holder. To view a copy of this licence, visit http://creativecomm ons.org/licenses/by/4.0/.

Funded by $\mathrm{SCOAP}^{3}$.

\section{Appendix: The derivation of embedding condition from the Gauss equations}

We take the nonzero independent components of the Riemann tensor for the generalized Vaidya metric (4) as

$$
\begin{aligned}
& R_{1212}=0 \\
& R_{1220}=\frac{r m^{\prime}-m}{r}, \\
& R_{1010}=-\frac{r^{2} m^{\prime \prime}-2 r m^{\prime}+2 m}{r^{3}}, \\
& R_{2323}=2 r m \sin ^{2} \theta, \\
& R_{2020}=\frac{1}{r^{2}}\left(2 r m m^{\prime}-r^{2} m^{\prime}-r^{2} \dot{m}-2 m^{2}+r m\right) .
\end{aligned}
$$

The other nonzero components can be written in terms of $R_{1220}, R_{1010}, R_{2323}$ and $R_{2020}$. Substituting all components of $R_{\mu \nu \delta \gamma}$ in the Gauss equations (15a) we obtain a system of equations in terms of the components of the symmetric tensor $b_{\mu \nu}$. These are given by

$$
\begin{aligned}
& 0=b_{11} b_{22}-b_{12} b_{12}, \\
& 0=b_{11} b_{23}-b_{13} b_{12},
\end{aligned}
$$




$$
\begin{aligned}
0 & =b_{11} b_{20}-b_{10} b_{12}, \\
0 & =b_{12} b_{23}-b_{13} b_{22}, \\
R_{1220} & =b_{12} b_{20}-b_{10} b_{22}, \\
0 & =b_{13} b_{20}-b_{10} b_{23}, \\
0 & =b_{11} b_{33}-b_{13} b_{13}, \\
0 & =b_{11} b_{30}-b_{10} b_{13}, \\
0 & =b_{12} b_{33}-b_{13} b_{23}, \\
0 & =b_{12} b_{30}-b_{10} b_{23}, \\
R_{1220} \sin ^{2} \theta & =b_{13} b_{30}-b_{10} b_{33}, \\
R_{1010} & =b_{11} b_{00}-b_{10} b_{10}, \\
0 & =b_{12} b_{30}-b_{13} b_{20}, \\
0 & =b_{12} b_{00}-b_{10} b_{20}, \\
0 & =b_{13} b_{00}-b_{10} b_{30}, \\
R_{2323} & =b_{22} b_{33}-b_{23} b_{23}, \\
0 & =b_{22} b_{30}-b_{20} b_{23}, \\
0 & =b_{23} b_{30}-b_{20} b_{33}, \\
R_{2020} 0 & =b_{22} b_{00}-b_{20} b_{20}, \\
0 & =b_{23} b_{00}-b_{20} b_{30}, \\
\sin ^{2} \theta & =b_{33} b_{00}-b_{30} b_{30},
\end{aligned}
$$

This is a complicated system but we can simplify it. Equations (A.6)-(A.9), (A.11)-(A.15), (A.18)-(A.20), (A.22), (A.23) and (A.25) generate the following reductions

$$
\begin{aligned}
& b_{11}=0, \\
& b_{12}=0, \\
& b_{13}=0, \\
& b_{23}=0, \\
& b_{20}=0, \\
& b_{30}=0 .
\end{aligned}
$$

Now substituting (A.27)-(A.32) into (A.10), (A.16), (A.17), (A.21), (A.24) and (A.26) gives the following components of the Riemann tensor

$$
\begin{aligned}
R_{1220} & =-b_{10} b_{22}, \\
R_{1010} & =-b_{10}^{2}, \\
R_{2323} & =b_{22} b_{33}, \\
R_{2020} & =b_{22} b_{00}, \\
R_{1220} \sin ^{2} \theta & =-b_{10} b_{33}, \\
R_{2020} \sin ^{2} \theta & =b_{33} b_{00} .
\end{aligned}
$$

From (A.33)-(A.38) we can generate the condition

$$
R_{1220}=-\frac{\sqrt{-R_{1010} R_{2323}}}{\sin \theta},
$$

which can be written as

$R_{1220} R_{1330}+R_{1010} R_{2323}=0$,

since $R_{1220} \sin ^{2} \theta=R_{1330}$. In the above we have shown that using the components of the symmetric tensor $b_{\mu \nu}$ we obtained a restriction on components of the curvature tensor.

It is also possible to find the symmetric tensor $b_{\mu \nu}$ in terms of the curvature. Equations (A.33)-(A.36) and (A.38) can be solved in general in terms of components of the Riemann tensor. We obtain

$b_{11}=0$,

$b_{22}=\frac{\sqrt{R_{2323}}}{\sin \theta}$,

$b_{33}=\sqrt{R_{2323}} \sin \theta$,

$b_{00}=\frac{R_{2020} \sin \theta}{\sqrt{R_{2323}}}$,

$b_{10}=-\frac{R_{1220}}{\sqrt{R_{2323}}}$.

Finally note that the Codazzi equations (15b) do not provide any new information and lead to the same expression as in (A.39) because the Codazzi equations in this case are a consequence of the Gauss equations and Bianchi identities [57].

\section{References}

1. L.P. Eisenhart, Riemannian Geometry (Princeton University Press, Princeton, 1997)

2. J. Rosen, Rev. Mod. Phys. 37, 204 (1965)

3. H. Stephani, Commun. Math. Phys. 4, 137 (1967)

4. C.D. Collinson, J. Math. Phys. 9, 403 (1968)

5. H. Stephani, D. Kramer, M.A.H. MacCallum, C. Hoenselaers, E. Herlt, Exact Solutions of Einstein's Field Equations (Cambridge University Press, Cambridge, 2003)

6. M. Pavsic, The Landscape of Theoretical Physics: A Global View: From Point Particles to the Brane World and Beyond in Search of a Unifying Principle (Kluwer, Tranbjerg, 2001)

7. K. Karmarkar, Proc. Indian Acad. Sci. A 27, 56 (1948)

8. S.K. Maurya, M. Govender, Eur. Phys. J. C 77, 347 (2017)

9. S.K. Maurya, Y.K. Gupta, S. Ray, D. Deb, Eur. Phys. J. C 76, 693 (2016)

10. S.K. Maurya, Y.K. Gupta, S. Ray, D. Deb, Eur. Phys. J. C 77, 45 (2017)

11. K.N. Singh, P. Bhar, F. Rahaman, N. Pant, M. Rahaman, Int. J. Mod. Phys. A 32, 1750093 (2017)

12. P.F.K. Kuhfittig, Ann. Phys. 392, 63 (2018)

13. P.K.F. Kuhfittig, Pramana J. Phys. 92, 75 (2019)

14. P. Bhar, S.K. Maurya, Y.K. Gupta, T. Manna, Eur. Phys. J. A 52, $312(2016)$

15. S.K. Maurya, Y.K. Gupta, T.T. Smitha, F. Rahaman, Eur. Phys. J. A 52, 191 (2016)

16. K.N. Singh, N. Pant, M. Govender, Eur. Phys. J. C 77, 100 (2017)

17. K.N. Singh, M.H. Murad, N. Pant, Eur. Phys. J. A 53, 21 (2017)

18. P. Bhar, K.N. Singh, T. Manna, Int. J. Mod. Phys. D 26, 1750090 (2017) 
19. S.K. Maurya, Y.K. Gupta, F. Rahaman, M. Rahaman, A. Banerjee, Ann. Phys. 385, 532 (2017)

20. P. Bhar, M. Govender, Int. J. Mod. Phys. D 26, 1750053 (2017)

21. P. Fuloria, Astrophys. Space Sci. 362, 217 (2017)

22. F. Tello-Ortiz, S.K. Maurya, Y. Gomez-Leyton, Eur. Phys. J. C 80, $324(2020)$

23. S. Gedela, R.K. Bisht, N. Pant, Mod. Phys. Lett. A 34, 1950157 (2019)

24. A.K. Prasad, J. Kumar, S.K. Maurya, B. Dayanandan, Astrophys. Space Sci. 364, 66 (2019)

25. B. Dayanand, T.T. Smitha, S.K. Maurya, Astrophys. Space Sci. 365, 20 (2020)

26. M.K. Jasim, S.K. Maurya, A.S.M. Al-Sawaii, Astrophys. Space Sci. 365, 9 (2020)

27. K.N. Singh, S.K. Maurya, A. Errehymy, F. Rahaman, M. Daoud, Phys. Dark Univ. 30, 100620 (2020)

28. M. Govender, A. Maharaj, K.N. Singh, N. Pant, Mod. Phys. Lett. A 35, 2050164 (2020)

29. K.N. Singh, R.K. Bisht, S.K. Maurya, N. Pant, Chin. Phys. C 44, $035101(2020)$

30. M.H. Murad, Eur. Phys. J. C 78, 285 (2018)

31. N.F. Naidu, M. Govender, S.D. Maharaj, Eur. Phys. J. C 78, 48 (2018)

32. E. Kasner, Am. J. Math. 43, 130 (1921)

33. P.C. Vaidya, Proc. Indian Acad. Sci. A 33, 264 (1951)

34. S.D. Maharaj, G. Govender, M. Govender, Gen. Relativ. Gravit. 44, 1089 (2012)

35. K.S. Govinder, M. Govender, Gen. Relativ. Gravit. 44, 147 (2012)

36. K.P. Reddy, M. Govender, S.D. Maharaj, Gen. Relativ. Gravit. 47, 35 (2015)

37. S. Das, R. Sharma, B.C. Paul, R. Deb, Astrophys. Space Sci. 361, 99 (2016)
38. G. Govender, B.P. Brassel, S.D. Maharaj, Eur. Phys. J. C 75, 324 (2015)

39. N.F. Naidu, M. Govender, S. Thirukkanesh, S.D. Maharaj, Gen. Relativ. Gravit. 49, 95 (2017)

40. B.P. Brassel, R. Goswami, S.D. Maharaj, Phys. Rev. D 95, 124051 (2017)

41. V. Husain, Phys. Rev. D 53, R1759 (1996)

42. E.N. Glass, J.P. Krisch, Phys. Rev. D 57, R5945 (1998)

43. C.E. Weatherburn, An Introduction to Riemannian Geometry (Cambridge University Press, Cambridge, 2008)

44. F. Schur, Math. Ann. 27, 163 (1886)

45. S.N. Pandey, S.P. Sharma, Gen. Relativ. Gravit. 14, 113 (1977)

46. A. Wang, Y. Wu, Gen. Relativ. Gravit. 31, 107 (1999)

47. E.N. Glass, J.P. Krisch, Class. Quantum Grav. 16, 1175 (1999)

48. A.K. Dawood, S.G. Ghosh, Phys. Rev. D 70, 104010 (2004)

49. S.G. Ghosh, A.K. Dawood, Gen. Relativ. Gravit. 40, 9 (2008)

50. B.P. Brassel, S.D. Maharaj, R. Goswami, Gen. Relativ. Gravit. 49, 101 (2017)

51. M. Govender, Int. J. Mod. Phys. D 22, 1350049 (2013)

52. G.Z. Abebe, S.D. Maharaj, Eur. Phys. J. C 79, 849 (2019)

53. R. Mohanlal, R. Narain, S.D. Maharaj, J. Math. Phys. 58, 072503 (2017)

54. A.B. Mahomed, S.D. Maharaj, R. Narain, AIP Adv. 10, 035208 (2020)

55. A.B. Mahomed, S.D. Maharaj, R. Narain, Eur. Phys. J. Plus 134, 545 (2019)

56. A.B. Mahomed, S.D. Maharaj, R. Narain, Eur. Phys. J. Plus 135, $351(2020)$

57. H.F. Goenner, Gen. Relativ. Gravit. 8, 139 (1977) 